DESY 97-257

hep-ph/9801380

December 1997

\title{
Two-loop three-gluon vertex in zero-momentum limit
}

\author{
A. I. Davydychev ${ }^{a, b, \text {, }, ~ P . ~ O s l a n d ~}{ }^{a, c, \text { P }}$ and O. V. Tarasov ${ }^{d, \beta}$ \\ ${ }^{a}$ Department of Physics, University of Bergen, \\ Allégaten 55, N-5007 Bergen, Norway \\ ${ }^{b}$ Institute for Nuclear Physics, Moscow State University, \\ 119899, Moscow, Russia \\ ${ }^{c}$ Deutsches Elektronen-Synchrotron DESY, D-22603 Hamburg, Germany \\ ${ }^{d}$ IfH, DESY-Zeuthen, Platanenallee 6, D-15738 Zeuthen, Germany
}

\begin{abstract}
The two-loop three-gluon vertex is calculated in an arbitrary covariant gauge, in the limit when one of the external momenta vanishes. The differential WardSlavnov-Taylor (WST) identity related to this limit is discussed, and the relevant results for the ghost-gluon vertex and two-point functions are obtained. Together with the differential WST identity, they provide another independent way for calculating the three-gluon vertex. The renormalization of the results obtained is also presented.
\end{abstract}

\footnotetext{
1davyd@theory.npi.msu.su

${ }^{2}$ Per.Osland@fi.uib.no

3 tarasov@ifh.de. On leave from Joint Institute for Nuclear Research, 141980, Dubna, Russia.
} 


\section{Introduction}

Jet studies are becoming increasingly precise, both as a testing ground for QCD, and as a background for new physics (e.g. Higgs searches). Increasing precision, among other things, requires knowledge of the fundamental QCD vertices to higher loops.

The one-loop vertices have been known for quite some time. Celmaster and Gonsalves presented in 1979 [1] the one-loop result for the three-gluon vertex, for off-shell gluons, restricted to the symmetric case, $p_{1}^{2}=p_{2}^{2}=p_{3}^{3}$, in an arbitrary covariant gauge. The result of [1] was confirmed by Pascual and Tarrach [2]. Ball and Chiu then in 1980 considered the general off-shell case, but restricted to the Feynman gauge [3]. Later, various on-shell results have also been given, by Brandt and Frenkel [4, restricted to the infrared-singular parts only (in an arbitrary covariant gauge), and by Nowak, Praszałowicz and Słomiński [5], who also gave the finite parts for the case of two gluons being on-shell (in Feynman gauge). The most general results, valid for arbitrary values of the space-time dimension and the covariant-gauge parameter, have been presented in our previous paper [6]. Some results for the one-loop quark-gluon vertex (or its Abelian part which is related to the QED vertex) can be found in [7].

The present paper is devoted to a study of two-loop corrections to the three-gluon vertex in the zero-momentum limit. This limit refers to the case when one gluon has vanishing momentum. The remaining two momenta must then be equal and opposite, so there is only one dimensionful scale, $p^{2}$. In this limit, the renormalized expressions for QCD vertices in the Feynman gauge have been presented by Braaten and Leveille [8]. Information about Green functions is also required for calculation of certain quantities related to the renormalization group equations, such as the $\beta$ function and anomalous dimensions. The two-loop-order contributions to these quantities were calculated in refs. [9, 10, 11, 12], whereas the three-loop-order results were obtained in [13, 14]. Moreover, recently the four-loop-order expressions became available [15].

When massless quarks are considered, the scalar functions corresponding to the coefficients of different tensor structures are in the zero-momentum limit rather simple: apart from non-trivial coefficients, they are given by $p^{2}$ raised to some power (determined by the dimension of space-time). Also, the tensorial structure is considerably simpler than in the general case. Although the zero-momentum limit has limited physical applications, it serves as an important reference point, against which more general results can be checked.

With one gluon momentum vanishing, there are two Ward-Slavnov-Taylor (WST) identities, one corresponding to the vanishing momentum, and one corresponding to the finite momentum. The identity corresponding to the vanishing momentum turns out to be a differential identity. In this case, the three-gluon vertex can actually be completely constructed from the two-point functions and the ghost-gluon vertex, with no additional transverse term.

In the present paper, we realize two ways to calculate the two-loop three-gluon vertex in an arbitrary covariant gauge. One of them is a straightforward calculation of all diagrams contributing to the three-gluon vertex at this order. Another way is based on using the results for the ghost-gluon vertex and the two-point functions, together with the corresponding WST identities. The renormalized expressions are also obtained. 


\section{Preliminaries}

The lowest-order gluon propagator is

$$
\delta^{a_{1} a_{2}} \frac{1}{p^{2}}\left(g_{\mu_{1} \mu_{2}}-\xi \frac{p_{\mu_{1}} p_{\mu_{2}}}{p^{2}}\right)
$$

where $\xi \equiv 1-\alpha$ is the gauge parameter corresponding to a general covariant gauge, defined such that $\xi=0(\alpha=1)$ is the Feynman gauge. Here and henceforth, a causal prescription is understood, $1 / p^{2} \rightarrow 1 /\left(p^{2}+\mathrm{i} 0\right)$.

The three-gluon vertex is defined as

$$
\Gamma_{\mu_{1} \mu_{2} \mu_{3}}^{a_{1} a_{2} a_{3}}\left(p_{1}, p_{2}, p_{3}\right) \equiv-\mathrm{i} g f^{a_{1} a_{2} a_{3}} \Gamma_{\mu_{1} \mu_{2} \mu_{3}}\left(p_{1}, p_{2}, p_{3}\right)
$$

where $f^{a_{1} a_{2} a_{3}}$ are the totally antisymmetric colour structures corresponding to the adjoint representation of the gauge group (for example, $\mathrm{SU}(N)$ or any other semi-simple gauge group). In fact, also completely symmetric colour structures $d^{a_{1} a_{2} a_{3}}$ might be considered, but they do not appear in the perturbative calculation of QCD three-point vertices at the one- and two-loop level. Since the gluons are bosons, and since the colour structures $f^{a_{1} a_{2} a_{3}}$ are antisymmetric, $\Gamma_{\mu_{1} \mu_{2} \mu_{3}}\left(p_{1}, p_{2}, p_{3}\right)$ must also be antisymmetric under any interchange of a pair of gluon momenta and the corresponding Lorentz indices.

When one of the momenta is zero, the three-gluon vertex contains only two tensor structures!,

$\Gamma_{\mu_{1} \mu_{2} \mu_{3}}(p,-p, 0)=\left(2 g_{\mu_{1} \mu_{2}} p_{\mu_{3}}-g_{\mu_{1} \mu_{3}} p_{\mu_{2}}-g_{\mu_{2} \mu_{3}} p_{\mu_{1}}\right) T_{1}\left(p^{2}\right)-p_{\mu_{3}}\left(g_{\mu_{1} \mu_{2}}-\frac{p_{\mu_{1}} p_{\mu_{2}}}{p^{2}}\right) T_{2}\left(p^{2}\right)$.

In this decomposition, we basically adopt the notation of [8] for the scalar functions $T_{i}\left(p^{2}\right)$. The first tensor structure on the r.h.s. of eq. (2.3) corresponds to the lowest-order vertex. There is the following correspondence between the functions $T_{i}$ and the scalar functions $A$ and $C$ used in [3] (cf. also in [6]):

$$
T_{1}\left(p^{2}\right) \leftrightarrow A\left(p^{2}, p^{2} ; 0\right), \quad T_{2}\left(p^{2}\right) \leftrightarrow-2 p^{2} C\left(p^{2}, p^{2} ; 0\right) .
$$

At the lowest, "zero-loop" order, the Yang-Mills term of the QCD Lagrangian yields"

$$
T_{1}^{(0)}=1, \quad T_{2}^{(0)}=0 .
$$

For a quantity $X$ (e.g. any of the scalar functions contributing to the propagators or the vertices), we shall denote the zero-loop-order contribution as $X^{(0)}$ (cf. eq. (2.5)), the one-loop-order contribution as $X^{(1)}$, and the two-loop-order contribution as $X^{(2)}$. In this paper, as a rule,

$$
X^{(L)}=X^{(L, \xi)}+X^{(L, q)}
$$

where $X^{(L, \xi)}$ denotes the contribution of gluon and ghost loops in a general covariant gauge (2.1) (in particular, $X^{(L, 0)}$ corresponds to the Feynman gauge, $\xi=0$ ), while $X^{(L, q)}$ represents the contribution of the quark loops.

\footnotetext{
${ }^{1}$ This is a corollary of the differential WST identity, see in section 3 .

${ }^{2}$ We include the contribution $T_{1}^{(0)}=1$ into the definition of $T_{1}\left(p^{2}\right)$, eq. (2.3).
} 
The ghost-gluon vertex can be represented as

$$
\widetilde{\Gamma}_{\mu_{3}}^{a_{1} a_{2} a_{3}}\left(p_{1}, p_{2} ; p_{3}\right) \equiv-\mathrm{i} g f^{a_{1} a_{2} a_{3}} p_{1}^{\mu} \widetilde{\Gamma}_{\mu \mu_{3}}\left(p_{1}, p_{2} ; p_{3}\right)
$$

where $p_{1}$ is the out-ghost momentum, $p_{2}$ is the in-ghost momentum, $p_{3}$ and $\mu_{3}$ are the momentum and the Lorentz index of the gluon (all momenta are ingoing). For $\widetilde{\Gamma}_{\mu \mu_{3}}$, the following decomposition was used in [3]:

$$
\begin{aligned}
\widetilde{\Gamma}_{\mu \mu_{3}}\left(p_{1}, p_{2} ; p_{3}\right)=g_{\mu \mu_{3}} a\left(p_{3}, p_{2}, p_{1}\right) & -p_{3 \mu} p_{2 \mu_{3}} b\left(p_{3}, p_{2}, p_{1}\right)+p_{1_{\mu}} p_{3 \mu_{3}} c\left(p_{3}, p_{2}, p_{1}\right) \\
& +p_{3 \mu} p_{1_{\mu_{3}}} d\left(p_{3}, p_{2}, p_{1}\right)+p_{1_{\mu}} p_{1_{\mu_{3}}} e\left(p_{3}, p_{2}, p_{1}\right)
\end{aligned}
$$

At the "zero-loop" level,

$$
\widetilde{\Gamma}_{\mu \mu_{3}}^{(0)}=g_{\mu \mu_{3}},
$$

and therefore all the scalar functions involved in (2.8) vanish at this order, except one, $a^{(0)}=1$.

We shall need the results for the ghost-gluon vertex (2.8) for two different configurations: (i) when the gluon momentum, $p_{3}$, is zero and (ii) when the in-ghost momentum, $p_{2}$, is zero. In the former case, we get

$$
\widetilde{\Gamma}_{\mu \mu_{3}}(-p, p ; 0)=g_{\mu \mu_{3}} a_{3}\left(p^{2}\right)+p_{\mu} p_{\mu_{3}} e_{3}\left(p^{2}\right), \quad a_{3}\left(p^{2}\right) \equiv a(0, p,-p), \quad e_{3}\left(p^{2}\right) \equiv e(0, p,-p),
$$

whereas in the latter case we obtain

$$
\widetilde{\Gamma}_{\mu \mu_{3}}(p, 0 ;-p)=g_{\mu \mu_{3}} a_{2}\left(p^{2}\right)+p_{\mu} p_{\mu_{3}} e_{2}^{\prime}\left(p^{2}\right), \quad a_{2}\left(p^{2}\right) \equiv a(-p, 0, p), \quad e_{2}^{\prime}\left(p^{2}\right) \equiv e^{\prime}(-p, 0, p),
$$

with

$$
e^{\prime}\left(p_{3}, p_{2}, p_{1}\right) \equiv e\left(p_{3}, p_{2}, p_{1}\right)-c\left(p_{3}, p_{2}, p_{1}\right)-d\left(p_{3}, p_{2}, p_{1}\right)
$$

We shall also denote

$$
d_{2}\left(p^{2}\right) \equiv d(-p, 0, p)
$$

We do not need to consider $\widetilde{\Gamma}_{\mu \mu_{3}}(0, p,-p)\left(p_{1}=0\right)$ because it does not enter the WST identities (see in section 3). Moreover, the proper ghost-gluon vertex (2.7) vanishes in this limit, for it contains $p_{1}{ }^{\mu}$.

The gluon polarization operator is defined as

$$
\Pi_{\mu_{1} \mu_{2}}^{a_{1} a_{2}}(p) \equiv-\delta^{a_{1} a_{2}}\left(p^{2} g_{\mu_{1} \mu_{2}}-p_{\mu_{1}} p_{\mu_{2}}\right) J\left(p^{2}\right)
$$

while the ghost self energy is

$$
\widetilde{\Pi}^{a_{1} a_{2}}\left(p^{2}\right)=\delta^{a_{1} a_{2}} p^{2}\left[G\left(p^{2}\right)\right]^{-1} .
$$

In the lowest-order approximation $J^{(0)}=G^{(0)}=1$.

\footnotetext{
${ }^{3}$ There was a misprint in eq. (2.8) of [6]: $G\left(p^{2}\right)$ should read $\left[G\left(p^{2}\right)\right]^{-1}$.
} 


\section{$3 \quad$ WST identity in the zero-momentum limit}

In a covariant gauge, the Ward-Slavnov-Taylor (WST) identity [16 for the three-gluon vertex is of the following form (see e.g. in [17]):

$$
\begin{aligned}
p_{3}^{\mu_{3}} \Gamma_{\mu_{1} \mu_{2} \mu_{3}}\left(p_{1}, p_{2}, p_{3}\right)= & -J\left(p_{1}^{2}\right) G\left(p_{3}^{2}\right)\left(g_{\mu_{1}}^{\mu_{3}} p_{1}^{2}-p_{\mu_{\mu_{1}}} p_{1}{ }^{\mu_{3}}\right) \widetilde{\Gamma}_{\mu_{3} \mu_{2}}\left(p_{1}, p_{3} ; p_{2}\right) \\
& +J\left(p_{2}^{2}\right) G\left(p_{3}^{2}\right)\left(g_{\mu_{2}}{ }^{\mu_{3}} p_{2}^{2}-p_{2 \mu_{2}} p_{2}{ }^{\mu_{3}}\right) \widetilde{\Gamma}_{\mu_{3} \mu_{1}}\left(p_{2}, p_{3} ; p_{1}\right) .
\end{aligned}
$$

It is easy to see that the $c$ and $e$ functions from the ghost-gluon vertex (2.8) do not contribute to this identity.

Consider what follows from (3.1) in the limit when one of the momenta vanishes. We should distinguish between two different cases: when the vanishing momentum is the one with which the three-gluon vertex is contracted, and when it is not. In the former case, we obtain a differential identity, whereas in the latter case we get an ordinary identity.

In the differential case, we should consider $p_{3} \equiv \delta \rightarrow 0, p_{1} \equiv p, p_{2}=-p-\delta$. We do not need the terms of order $\delta^{2}$ and higher. In particular, $G\left(\delta^{2}\right)=G(0)+\mathcal{O}\left(\delta^{2}\right)$ and, for massless quarks, $G(0)=1$. When we expand the r.h.s. of eq. (3.1) in $\delta$, the lowest ("constant") term disappears, so only the term linear in $\delta$ is relevant. Differentiating both sides with respect to $\delta^{\mu_{3}}$ and putting $\delta=0$, we get

$$
\begin{aligned}
& \Gamma_{\mu_{1} \mu_{2} \mu_{3}}(p,-p, 0)=\left(2 g_{\mu_{1} \mu_{2}} p_{\mu_{3}}-g_{\mu_{1} \mu_{3}} p_{\mu_{2}}-g_{\mu_{2} \mu_{3}} p_{\mu_{1}}\right)\left[a_{2}\left(p^{2}\right)-p^{2} d_{2}\left(p^{2}\right)\right] J\left(p^{2}\right) G(0) \\
& +2 p_{\mu_{3}}\left(g_{\mu_{1} \mu_{2}}-\frac{p_{\mu_{1}} p_{\mu_{2}}}{p^{2}}\right)\left[\left(p^{2} d_{2}\left(p^{2}\right)+\widetilde{a}_{2}\left(p^{2}\right)-p^{2} \frac{\mathrm{d} a_{2}\left(p^{2}\right)}{\mathrm{d} p^{2}}\right) J\left(p^{2}\right)+p^{2} a_{2}\left(p^{2}\right) \frac{\mathrm{d} J\left(p^{2}\right)}{\mathrm{d} p^{2}}\right] G(0),
\end{aligned}
$$

where the functions $a_{2}\left(p^{2}\right)$ and $d_{2}\left(p^{2}\right)$ are defined in eqs. (2.11) and (2.13), respectively. The function $\widetilde{a}_{2}\left(p^{2}\right)$ is defined as

$$
\left.\widetilde{a}_{2}\left(p^{2}\right) \equiv p_{1_{\sigma}} \frac{\partial}{\partial p_{1_{\sigma}}} a\left(p_{3},-p_{1}-p_{3}, p_{1}\right)\right|_{p_{1}=-p_{3}=p} .
$$

It can be calculated directly at the diagrammatic level (see in section 5).

Considering contraction with a non-zero momentum, we get from eq. (3.1)

$$
p^{\mu_{1}} \Gamma_{\mu_{1} \mu_{2} \mu_{3}}(p,-p, 0)=-J\left(p^{2}\right) G\left(p^{2}\right) a_{3}\left(p^{2}\right)\left(g_{\mu_{2} \mu_{3}} p^{2}-p_{\mu_{2}} p_{\mu_{3}}\right),
$$

where $a_{3}\left(p^{2}\right)$ is defined in eq. (2.10). Contracting eq. (3.2) with $p^{\mu_{1}}$ we get a different representation which should be equal to the r.h.s. of eq. (3.4). Therefore, the following relation should hold:

$$
G(0)\left[a_{2}\left(p^{2}\right)-p^{2} d_{2}\left(p^{2}\right)\right]=G\left(p^{2}\right) a_{3}\left(p^{2}\right) .
$$

Using eq. (3.5), the differential WST identity (3.2) can be re-written in a way which involves just the $a$ functions from the ghost-gluon vertex:

$$
\begin{aligned}
& \Gamma_{\mu_{1} \mu_{2} \mu_{3}}(p,-p, 0)=-\left[p_{\mu_{1}}\left(g_{\mu_{2} \mu_{3}}-\frac{p_{\mu_{2}} p_{\mu_{3}}}{p^{2}}\right)+p_{\mu_{2}}\left(g_{\mu_{1} \mu_{3}}-\frac{p_{\mu_{1}} p_{\mu_{3}}}{p^{2}}\right)\right] a_{3}\left(p^{2}\right) G\left(p^{2}\right) J\left(p^{2}\right) \\
& +2 p_{\mu_{3}}\left(g_{\mu_{1} \mu_{2}}-\frac{p_{\mu_{1}} p_{\mu_{2}}}{p^{2}}\right) G(0)\left[a_{2}\left(p^{2}\right) \frac{\mathrm{d}}{\mathrm{d} p^{2}}\left(p^{2} J\left(p^{2}\right)\right)-p^{2} J\left(p^{2}\right) \frac{\mathrm{d} a_{2}\left(p^{2}\right)}{\mathrm{d} p^{2}}+\widetilde{a}_{2}\left(p^{2}\right) J\left(p^{2}\right)\right] .
\end{aligned}
$$


For the scalar functions $T_{i}\left(p^{2}\right)$, the WST identity gives

$$
\begin{gathered}
T_{1}\left(p^{2}\right)=a_{3}\left(p^{2}\right) G\left(p^{2}\right) J\left(p^{2}\right), \\
T_{2}\left(p^{2}\right)=2 T_{1}\left(p^{2}\right)-2 G(0)\left[a_{2}\left(p^{2}\right) \frac{\mathrm{d}}{\mathrm{d} p^{2}}\left(p^{2} J\left(p^{2}\right)\right)-p^{2} J\left(p^{2}\right) \frac{\mathrm{d} a_{2}\left(p^{2}\right)}{\mathrm{d} p^{2}}+\widetilde{a}_{2}\left(p^{2}\right) J\left(p^{2}\right)\right] .
\end{gathered}
$$

Therefore, the differential WST identity makes it possible to define the whole threegluon vertex (not only its longitudinal part) in terms of two-point functions and the ghost-gluon vertex. Moreover, it can be used as another independent way, in addition to the direct calculation, to obtain results for the three-gluon vertex.

\section{Results for the three-gluon vertex}

We shall use dimensional regularization [18], with the space-time dimension $n=4-2 \varepsilon$. The results for unrenormalized one-loop contributions to the scalar functions $T_{1}\left(p^{2}\right)$ and $T_{2}\left(p^{2}\right)$ (in arbitrary space-time dimension) can be found in ref. [6], eqs. (4.30), (4.31), (4.33) and (4.34). Expanding them in $\varepsilon$ we gett

$$
\begin{gathered}
T_{1}^{(1, \xi)}\left(p^{2}\right)=C_{A} \frac{g^{2} \eta}{(4 \pi)^{n / 2}}\left(-p^{2}\right)^{-\varepsilon}\left\{\frac{1}{\varepsilon}\left(-\frac{2}{3}-\frac{3}{4} \xi\right)-\frac{35}{18}+\frac{1}{2} \xi-\frac{1}{4} \xi^{2}\right. \\
\left.+\varepsilon\left(-\frac{107}{27}+\xi-\frac{1}{2} \xi^{2}\right)\right\}+\mathcal{O}\left(\varepsilon^{2}\right), \\
T_{1}^{(1, q)}\left(p^{2}\right)=T \frac{g^{2} \eta}{(4 \pi)^{n / 2}}\left(-p^{2}\right)^{-\varepsilon}\left\{\frac{4}{3 \varepsilon}+\frac{20}{9}+\frac{112}{27} \varepsilon\right\}+\mathcal{O}\left(\varepsilon^{2}\right), \\
T_{2}^{(1, \xi)}\left(p^{2}\right)=C_{A} \frac{g^{2} \eta}{(4 \pi)^{n / 2}}\left(-p^{2}\right)^{-\varepsilon}\left\{-\frac{4}{3}-2 \xi+\frac{1}{4} \xi^{2}+\varepsilon\left(-\frac{26}{9}-\xi+\frac{1}{4} \xi^{2}\right)\right\}+\mathcal{O}\left(\varepsilon^{2}\right), \\
T_{2}^{(1, q)}\left(p^{2}\right)=T \frac{g^{2} \eta}{(4 \pi)^{n / 2}}\left(-p^{2}\right)^{-\varepsilon}\left\{\frac{8}{3}+\frac{40}{9} \varepsilon\right\}+\mathcal{O}\left(\varepsilon^{2}\right) .
\end{gathered}
$$

In these equations, we use the standard notation $C_{A}$ for the eigenvalue of the quadratic Casimir operator in the adjoint representation,

$$
f^{a c d} f^{b c d}=C_{A} \delta^{a b} \quad\left(C_{A}=N \text { for the } \mathrm{SU}(N) \text { group }\right) .
$$

Furthermore,

$$
T \equiv N_{f} T_{R}, \quad T_{R}=\frac{1}{8} \operatorname{Tr}(I)=\frac{1}{2},
$$

where $I$ is the "unity" in the space of Dirac matrices (we assume that $\operatorname{Tr}(I)=4$ ), $N_{f}$ is the number of quarks and

$$
\eta \equiv \frac{\Gamma^{2}\left(\frac{n}{2}-1\right)}{\Gamma(n-3)} \Gamma\left(3-\frac{n}{2}\right)=\frac{\Gamma^{2}(1-\varepsilon)}{\Gamma(1-2 \varepsilon)} \Gamma(1+\varepsilon)=e^{-\gamma \varepsilon}\left(1-\frac{1}{12} \pi^{2} \varepsilon^{2}+\mathcal{O}\left(\varepsilon^{3}\right)\right) .
$$

\footnotetext{
${ }^{4}$ In all unrenormalized expressions given in sections 4-7 and in Appendix A, the bare quantities $g^{2}=g_{B}^{2}$ and $\xi=\xi_{B}$ are understood, i.e. the same as those given in the lowest-order functions (2.1)-(2.2). When the renormalization is discussed, these bare quantities get a subscript " $B$ " (see in section 8).
} 
Here $\gamma \simeq 0.57721566 \ldots$ is the Euler constant. The $\varepsilon$ terms in the expressions (4.1)-(4.4) are needed when these expressions are multiplied by terms which diverge like $1 / \varepsilon$, e.g., for the calculation of reducible unrenormalized two-loop-order contributions. The $\varepsilon$ terms are also necessary for getting the renormalized two-loop-order results, see section 8 .

The diagrams contributing to the three-gluon vertex at the two-loop level are shown in Fig. 1 . Each diagram should be considered with two other "rotations", corresponding to permutations of the external legs. The grey blob corresponds to a sum of all one-loop contributions to the gluon polarization operator, including the gluon, ghost and quark loops insertions], cf. Fig. 2a of [6]. Note that non-planar graphs do not contribute to the two-loop vertex, since their over-all colour factors vanish, due to the Jacobi identity (cf. Fig. 6 of ref. [20] where this is explained).

When one external momentum vanishes, technically the problem reduces to the calculation of two-point two-loop Feynman integrals. To calculate the occurring integrals with higher powers of the propagators, the integration-by-parts procedure [21] has been used. For the integrals with numerators, some other known algorithms [21] (see also in [22]) were employed. Straightforward calculation of the sum of all these contributions] yields the following results for the unrenormalized scalar functions:

$$
\begin{gathered}
T_{1}^{(2, \xi)}\left(p^{2}\right)=C_{A}^{2} \frac{g^{4} \eta^{2}}{(4 \pi)^{n}}\left(-p^{2}\right)^{-2 \varepsilon}\left\{\frac{1}{\varepsilon^{2}}\left(-\frac{13}{8}-\frac{7}{16} \xi+\frac{15}{32} \xi^{2}\right)+\frac{1}{\varepsilon}\left(-\frac{311}{48}+\frac{13}{96} \xi-\frac{29}{48} \xi^{2}+\frac{7}{16} \xi^{3}\right)\right. \\
\left.-\frac{6965}{288}-\frac{1}{4} \zeta_{3}-\frac{509}{576} \xi+\frac{15}{8} \xi \zeta_{3}-\frac{115}{144} \xi^{2}+\frac{13}{16} \xi^{3}+\frac{1}{16} \xi^{4}\right\}+\mathcal{O}(\varepsilon), \\
T_{1}^{(2, q)}\left(p^{2}\right)=C_{A} T \frac{g^{4} \eta^{2}}{(4 \pi)^{n}}\left(-p^{2}\right)^{-2 \varepsilon}\left\{\frac{1}{\varepsilon^{2}}\left(\frac{5}{2}-\xi\right)+\frac{1}{\varepsilon}\left(\frac{97}{12}-\frac{1}{3} \xi-\frac{2}{3} \xi^{2}\right)\right. \\
\left.+\frac{1675}{72}+8 \zeta_{3}+\frac{16}{9} \xi-\frac{22}{9} \xi^{2}\right\} \\
+C_{F} T \frac{g^{4} \eta^{2}}{(4 \pi)^{n}}\left(-p^{2}\right)^{-2 \varepsilon}\left\{\frac{2}{\varepsilon}+\frac{55}{3}-16 \zeta_{3}\right\}+\mathcal{O}(\varepsilon), \\
T_{2}^{(2, \xi)}\left(p^{2}\right)=C_{A}^{2} \frac{g^{4} \eta^{2}}{(4 \pi)^{n}}\left(-p^{2}\right)^{-2 \varepsilon}\left\{\frac{1}{\varepsilon}\left(-\frac{22}{3}-\frac{11}{6} \xi+\frac{8}{3} \xi^{2}-\frac{7}{16} \xi^{3}\right)\right. \\
\left.-\frac{1013}{36}-\zeta_{3}+\frac{13}{9} \xi-\frac{1}{2} \xi \zeta_{3}-\frac{83}{144} \xi^{2}+\frac{3}{4} \xi^{3}-\frac{1}{8} \xi^{4}\right\}+\mathcal{O}(\varepsilon), \\
T_{2}^{(2, q)}\left(p^{2}\right)=C_{A} T \frac{g^{4} \eta^{2}}{(4 \pi)^{n}}\left(-p^{2}\right)^{-2 \varepsilon}\left\{\frac{1}{\varepsilon}\left(\frac{32}{3}-\frac{16}{3} \xi+\frac{2}{3} \xi^{2}\right)+\frac{289}{9}-\frac{133}{18} \xi+\frac{4}{9} \xi^{2}\right\} \\
+8 C_{F} T \frac{g^{4} \eta^{2}}{(4 \pi)^{n}}\left(-p^{2}\right)^{-2 \varepsilon}+\mathcal{O}(\varepsilon),
\end{gathered}
$$

\footnotetext{
${ }^{5}$ To produce the figures, the AXODRAW package [19] was used.

${ }^{6}$ Here and henceforth, we do not show contributions involving tadpole-like insertions which vanish in the framework of dimensional regularization $[18]$.

${ }^{7}$ For this calculation, two independent computer programs written in REDUCE [23] and FORM [24] were used.
} 
where $\zeta_{3} \equiv \zeta(3)=\sum_{j=1}^{\infty} j^{-3} \simeq 1.2020569 \ldots$ is the value of Riemann's zeta function; $C_{F}$ is the eigenvalue of the quadratic Casimir operator in the fundamental representation. For the $\mathrm{SU}(N)$ group, $C_{F}=\left(N^{2}-1\right) /(2 N)$.

\section{$5 \quad$ Results for the ghost-gluon vertex}

In order to check the WST identity, we need results for the ghost-gluon vertex in two limits corresponding to eqs. (2.10) and (2.11). We shall also need the derivative $\widetilde{a}_{2}\left(p^{2}\right)$, eq. (3.3).

The relevant one-loop results (for an arbitrary $n$ ) are listed in Appendix A. Expanding them in $\varepsilon$ we get

$$
\begin{gathered}
a_{3}^{(1)}\left(p^{2}\right)=C_{A} \frac{g^{2} \eta}{(4 \pi)^{n / 2}}\left(-p^{2}\right)^{-\varepsilon}(1-\xi)\left\{\frac{1}{2 \varepsilon}+\frac{1}{2}+\varepsilon\right\}+\mathcal{O}\left(\varepsilon^{2}\right), \\
a_{2}^{(1)}\left(p^{2}\right)=C_{A} \frac{g^{2} \eta}{(4 \pi)^{n / 2}}\left(-p^{2}\right)^{-\varepsilon}(1-\xi)\left\{\frac{1}{2 \varepsilon}+\frac{1}{4} \xi+\frac{1}{2} \xi \varepsilon\right\}+\mathcal{O}\left(\varepsilon^{2}\right), \\
\widetilde{a}_{2}^{(1)}\left(p^{2}\right)=C_{A} \frac{g^{2} \eta}{(4 \pi)^{n / 2}}\left(-p^{2}\right)^{-\varepsilon}\left\{\frac{1}{\varepsilon}\left(\frac{1}{2}+\frac{1}{4} \xi\right)+\frac{1}{4} \xi+\frac{1}{8} \xi^{2}+\varepsilon\left(1-\frac{1}{4} \xi+\frac{3}{8} \xi^{2}\right)\right\}+\mathcal{O}\left(\varepsilon^{2}\right), \\
p^{2} e_{3}^{(1)}\left(p^{2}\right)=C_{A} \frac{g^{2} \eta}{(4 \pi)^{n / 2}}\left(-p^{2}\right)^{-\varepsilon}\left\{\frac{1}{2}+\frac{1}{4} \xi+\varepsilon\right\}+\mathcal{O}\left(\varepsilon^{2}\right), \\
p^{2} e_{2}^{\prime(1)}\left(p^{2}\right)=C_{A} \frac{g^{2} \eta}{(4 \pi)^{n / 2}}\left(-p^{2}\right)^{-\varepsilon}(1-\xi)(2-\xi)\left\{\frac{1}{4}+\frac{1}{2} \varepsilon\right\}+\mathcal{O}\left(\varepsilon^{2}\right) .
\end{gathered}
$$

Two-loop contributions to the ghost-gluon vertex are shown in Fig. 2. As in the case of the three-gluon vertex (cf. Fig. 1), non-planar graphs do not contribute (cf. ref. [20]). Straightforward calculation gives the following results:

$$
\begin{gathered}
a_{3}^{(2, \xi)}\left(p^{2}\right)=C_{A}^{2} \frac{g^{4} \eta^{2}}{(4 \pi)^{n}}\left(-p^{2}\right)^{-2 \varepsilon}\left\{\frac{1}{\varepsilon^{2}}\left(\frac{5}{8}-\frac{7}{8} \xi+\frac{1}{4} \xi^{2}\right)+\frac{1}{\varepsilon}\left(\frac{13}{8}-\frac{35}{16} \xi+\frac{9}{16} \xi^{2}\right)\right. \\
\left.+\frac{257}{48}-\frac{1}{2} \zeta_{3}-\frac{635}{96} \xi-\frac{1}{8} \xi \zeta_{3}+\frac{23}{16} \xi^{2}+\frac{3}{16} \xi^{2} \zeta_{3}\right\}+\mathcal{O}(\varepsilon), \\
a_{3}^{(2, q)}\left(p^{2}\right)=\frac{1}{4} C_{A} T \frac{g^{4} \eta^{2}}{(4 \pi)^{n}}\left(-p^{2}\right)^{-2 \varepsilon}+\mathcal{O}(\varepsilon), \\
p^{2} e_{3}^{(2, \xi)}\left(p^{2}\right)=C_{A}^{2} \frac{g^{4} \eta^{2}}{(4 \pi)^{n}}\left(-p^{2}\right)^{-2 \varepsilon}\left\{\frac{1}{\varepsilon}\left(\frac{5}{2}+\frac{1}{2} \xi-\frac{1}{4} \xi^{2}\right)+\frac{65}{6}+\frac{1}{8} \zeta_{3}-\frac{11}{12} \xi+\frac{5}{16} \xi \zeta_{3}-\frac{3}{16} \xi^{2}\right\}+\mathcal{O}(\varepsilon), \\
a_{2}^{(2, \xi)}\left(p^{2}\right)=C_{A}^{2} \frac{g^{4} \eta^{2}}{(4 \pi)^{n}}\left(-p^{2}\right)^{-2 \varepsilon}(1-\xi)\left\{\frac{1}{\varepsilon^{2}}\left(\frac{5}{8}-\frac{1}{4} \xi\right)+\frac{1}{\varepsilon}\left(\frac{19}{24}+\frac{13}{48} \xi-\frac{3}{8} \xi^{2}\right)\right. \\
\left.+\frac{227}{72}-\zeta_{3}+\frac{53}{144} \xi-\frac{13}{16} \xi^{2}-\frac{1}{16} \xi^{3}\right\}+\mathcal{O}(\varepsilon),(5.10)
\end{gathered}
$$




$$
\begin{gathered}
a_{2}^{(2, q)}\left(p^{2}\right)=C_{A} T \frac{g^{4} \eta^{2}}{(4 \pi)^{n}}\left(-p^{2}\right)^{-2 \varepsilon}(1-\xi)^{2}\left\{-\frac{1}{3 \varepsilon}-\frac{11}{9}\right\}+\mathcal{O}(\varepsilon), \\
p^{2} e_{2}^{\prime(2, \xi)}\left(p^{2}\right)=C_{A}^{2} \frac{g^{4} \eta^{2}}{(4 \pi)^{n}}\left(-p^{2}\right)^{-2 \varepsilon}(1-\xi)\left\{\frac{1}{\varepsilon}\left(\frac{5}{6}-\frac{5}{6} \xi+\frac{3}{8} \xi^{2}\right)\right. \\
\left.+\frac{89}{36}+\frac{5}{8} \zeta_{3}-\frac{65}{36} \xi-\frac{3}{16} \xi \zeta_{3}+\frac{13}{16} \xi^{2}+\frac{1}{16} \xi^{3}\right\}+\mathcal{O}(\varepsilon), \\
p^{2} e_{2}^{\prime(2, q)}\left(p^{2}\right)=C_{A} T \frac{g^{4} \eta^{2}}{(4 \pi)^{n}}\left(-p^{2}\right)^{-2 \varepsilon}(1-\xi)^{2}\left\{\frac{1}{3 \varepsilon}+\frac{11}{9}\right\}+\mathcal{O}(\varepsilon) .
\end{gathered}
$$

The derivative (3.3) has been calculated in the following way. The momenta $p_{1}$ and $p_{3}$ are considered as independent variables, whereas $p_{2}=-p_{1}-p_{3}$. Therefore, the momentum $p_{1}$ flows from the in-ghost leg to the out-ghost leg. An unambiguous $p_{1}$ path inside the diagram can be chosen as the one coinciding with the ghost line. This is convenient, since all we need to differentiate are just two types of objects: ghost propagators and ghost-gluon vertices occurring along this path. In this way, we avoid differentiating gluon propagators and three-gluon vertices. We also avoid getting third powers of propagators.

Technically, this was realized as follows. The list of diagrams contributing to the ghost-gluon vertex, Fig. 2, was taken. Then, the propagators and vertices along the ghost path were "marked" by introducing an extra argument (say, z). Of course, the closed ghost loops should not be marked. Then, the derivative with respect to $z$ was considered, and the rules for differentiating the ghost-gluon vertex and the ghost propagator (with subsequent contraction with $p_{1_{\mu_{1}}}$ ) were supplied. It is very important that we do not really need expressions with different momenta; we just formally differentiate along the ghost line, and then perform all calculations for $p_{1}=-p_{3}=p, p_{2}=0$. Finally, extracting the coefficient of $g_{\mu \mu_{3}}$ gives the following results for the function (3.3):

$$
\begin{gathered}
\widetilde{a}_{2}^{(2, \xi)}\left(p^{2}\right)=C_{A}^{2} \frac{g^{4} \eta^{2}}{(4 \pi)^{n}}\left(-p^{2}\right)^{-2 \varepsilon}\left\{\frac{1}{\varepsilon^{2}}\left(\frac{3}{2}+\frac{5}{16} \xi-\frac{5}{32} \xi^{2}\right)+\frac{1}{\varepsilon}\left(\frac{121}{48}+\frac{185}{96} \xi+\frac{1}{24} \xi^{2}-\frac{7}{32} \xi^{3}\right)\right. \\
\left.+\frac{3085}{288}+\frac{1}{4} \zeta_{3}+\frac{1265}{576} \xi-\frac{7}{8} \xi \zeta_{3}+\frac{389}{288} \xi^{2}-\frac{13}{16} \xi^{3}-\frac{1}{32} \xi^{4}\right\}+\mathcal{O}(\varepsilon), \quad(5.14) \\
\widetilde{a}_{2}^{(2, q)}\left(p^{2}\right)=C_{A} T \frac{g^{4} \eta^{2}}{(4 \pi)^{n}}\left(-p^{2}\right)^{-2 \varepsilon}\left\{-\frac{1}{2 \varepsilon^{2}}+\frac{1}{\varepsilon}\left(-\frac{17}{12}-\frac{2}{3} \xi+\frac{1}{6} \xi^{2}\right)\right. \\
\left.-\frac{239}{72}-\frac{79}{36} \xi+\frac{7}{9} \xi^{2}\right\}+\mathcal{O}(\varepsilon) .
\end{gathered}
$$

\section{Results for the two-point functions}

Before presenting the results, let us make some general remarks. According to eq. (2.14), the gluon polarization operator is proportional to

$$
J\left(p^{2}\right)=1+J^{(1)}\left(p^{2}\right)+J^{(2)}\left(p^{2}\right)+\ldots
$$


Two-loop contributions to the gluon polarization operator are shown in Fig. 3. The gluon propagator is proportional to

$$
\frac{1}{J\left(p^{2}\right)}\left(g_{\mu_{1} \mu_{2}}-\frac{p_{\mu_{1}} p_{\mu_{2}}}{p^{2}}\right)+(1-\xi) \frac{p_{\mu_{1}} p_{\mu_{2}}}{p^{2}} .
$$

Therefore, the transverse part of the propagator is proportional to

$$
\left[J\left(p^{2}\right)\right]^{-1}=1-J^{(1)}\left(p^{2}\right)-J^{(2)}\left(p^{2}\right)+\left[J^{(1)}\left(p^{2}\right)\right]^{2}+\ldots
$$

According to eq. (2.15), the ghost propagator is proportional to

$$
G\left(p^{2}\right)=1+G^{(1)}\left(p^{2}\right)+G^{(2)}\left(p^{2}\right)+\ldots
$$

The ghost self energy (which is inverse to the propagator) is proportional to

$$
\begin{aligned}
{\left[G\left(p^{2}\right)\right]^{-1} } & =1-G^{(1)}\left(p^{2}\right)-G^{(2)(\text { irred })}\left(p^{2}\right)+\ldots \\
& =1-G^{(1)}\left(p^{2}\right)-G^{(2)}\left(p^{2}\right)+\left[G^{(1)}\left(p^{2}\right)\right]^{2}+\ldots
\end{aligned}
$$

Note that the one-loop contribution to the ghost self energy gives $-G^{(1)}\left(p^{2}\right)$. Two-loop contributions to the ghost self energy are shown in Fig. 4. They give $-G^{(2) \text { (irred) }}\left(p^{2}\right)$. According to eq. (6.5), the two-loop contribution to the ghost propagator consists of two parts, the irreducible one and the reducible one,

$$
G^{(2)}\left(p^{2}\right)=G^{(2) \text { (irred) }}\left(p^{2}\right)+G^{(2)(\text { red })}\left(p^{2}\right),
$$

where $G^{(2)(\text { red })}\left(p^{2}\right)=\left[G^{(1)}\left(p^{2}\right)\right]^{2}$.

One-loop results in arbitrary space-time dimension are available e.g. in [25, 6] (see also in Appendix A). When we expand them in $\varepsilon$ and keep the terms up to the order $\varepsilon$, we get

$$
\begin{gathered}
J^{(1, \xi)}\left(p^{2}\right)=C_{A} \frac{g^{2} \eta}{(4 \pi)^{n / 2}}\left(-p^{2}\right)^{-\varepsilon}\left\{\frac{1}{\varepsilon}\left(-\frac{5}{3}-\frac{1}{2} \xi\right)-\frac{31}{9}+\xi-\frac{1}{4} \xi^{2}\right. \\
\left.+\varepsilon\left(-\frac{188}{27}+2 \xi-\frac{1}{2} \xi^{2}\right)\right\}+\mathcal{O}\left(\varepsilon^{2}\right), \\
J^{(1, q)}\left(p^{2}\right)=T \frac{g^{2} \eta}{(4 \pi)^{n / 2}}\left(-p^{2}\right)^{-\varepsilon}\left\{\frac{4}{3 \varepsilon}+\frac{20}{9}+\frac{112}{27} \varepsilon\right\}+\mathcal{O}\left(\varepsilon^{2}\right), \\
G^{(1)}\left(p^{2}\right)=C_{A} \frac{g^{2} \eta}{(4 \pi)^{n / 2}}\left(-p^{2}\right)^{-\varepsilon}\left\{\frac{1}{\varepsilon}\left(\frac{1}{2}+\frac{1}{4} \xi\right)+1+2 \varepsilon\right\}+\mathcal{O}\left(\varepsilon^{2}\right) .
\end{gathered}
$$

Calculating the sum of one-particle irreducible two-loop diagrams contributing to the gluon polarization operator (shown in Fig. 3), we have obtained the following unrenormalized results:

$$
\begin{aligned}
J^{(2, \xi)}\left(p^{2}\right)=C_{A}^{2} \frac{g^{4} \eta^{2}}{(4 \pi)^{n}}( & \left.-p^{2}\right)^{-2 \varepsilon}\left\{\frac{1}{\varepsilon^{2}}\left(-\frac{25}{12}+\frac{5}{24} \xi+\frac{1}{4} \xi^{2}\right)+\frac{1}{\varepsilon}\left(-\frac{583}{72}+\frac{113}{144} \xi-\frac{19}{24} \xi^{2}+\frac{3}{8} \xi^{3}\right)\right. \\
& \left.-\frac{14311}{432}+\zeta_{3}+\frac{425}{864} \xi+2 \xi \zeta_{3}-\frac{71}{72} \xi^{2}+\frac{9}{16} \xi^{3}+\frac{1}{16} \xi^{4}\right\}+\mathcal{O}(\varepsilon),(6.10)
\end{aligned}
$$




$$
\begin{aligned}
J^{(2, q)}\left(p^{2}\right)= & C_{A} T \frac{g^{4} \eta^{2}}{(4 \pi)^{n}}\left(-p^{2}\right)^{-2 \varepsilon}\left\{\frac{1}{\varepsilon^{2}}\left(\frac{5}{3}-\frac{2}{3} \xi\right)+\frac{1}{\varepsilon}\left(\frac{101}{18}+\frac{8}{9} \xi-\frac{2}{3} \xi^{2}\right)\right. \\
& \left.+\frac{1961}{108}+8 \zeta_{3}+\frac{142}{27} \xi-\frac{22}{9} \xi^{2}\right\} \\
& +C_{F} T \frac{g^{4} \eta^{2}}{(4 \pi)^{n}}\left(-p^{2}\right)^{-2 \varepsilon}\left\{\frac{2}{\varepsilon}+\frac{55}{3}-16 \zeta_{3}\right\}+\mathcal{O}(\varepsilon) .
\end{aligned}
$$

Calculating the sum of the contributions (Fig. 4) to the ghost self energy (with a minus sign, cf. eq. (6.5)), we obtain

$$
\begin{aligned}
& G^{(2, \xi) \text { (irred) }}\left(p^{2}\right)=C_{A}^{2} \frac{g^{4} \eta^{2}}{(4 \pi)^{n}}\left(-p^{2}\right)^{-2 \varepsilon}\left\{\frac{1}{\varepsilon^{2}}\left(1+\frac{3}{16} \xi-\frac{3}{32} \xi^{2}\right)+\frac{1}{\varepsilon}\left(\frac{67}{16}-\frac{9}{32} \xi\right)\right. \\
& \left.+\frac{503}{32}-\frac{3}{4} \zeta_{3}-\frac{73}{64} \xi+\frac{3}{8} \xi^{2}-\frac{3}{16} \xi^{2} \zeta_{3}\right\}+\mathcal{O}(\varepsilon) \\
& G^{(2, q)}\left(p^{2}\right)=C_{A} T \frac{g^{4} \eta^{2}}{(4 \pi)^{n}}\left(-p^{2}\right)^{-2 \varepsilon}\left\{-\frac{1}{2 \varepsilon^{2}}-\frac{7}{4 \varepsilon}-\frac{53}{8}\right\}+\mathcal{O}(\varepsilon) .
\end{aligned}
$$

Note that there is no reducible part in $G^{(2, q)}$. The reducible part of $G^{(2, \xi)}$ is given by the square of eq. (6.9),

$$
G^{(2, \xi)(\mathrm{red})}\left(p^{2}\right)=C_{A}^{2} \frac{g^{4} \eta^{2}}{(4 \pi)^{n}}\left(-p^{2}\right)^{-2 \varepsilon}\left\{\frac{1}{\varepsilon^{2}}\left(\frac{1}{4}+\frac{1}{4} \xi+\frac{1}{16} \xi^{2}\right)+\frac{1}{\varepsilon}\left(1+\frac{1}{2} \xi\right)+3+\xi\right\}+\mathcal{O}(\varepsilon) .
$$

Therefore, using eq. (6.6) we get

$$
\begin{array}{r}
G^{(2, \xi)}\left(p^{2}\right)=C_{A}^{2} \frac{g^{4} \eta^{2}}{(4 \pi)^{n}}\left(-p^{2}\right)^{-2 \varepsilon}\left\{\frac{1}{\varepsilon^{2}}\left(\frac{5}{4}+\frac{7}{16} \xi-\frac{1}{32} \xi^{2}\right)+\frac{1}{\varepsilon}\left(\frac{83}{16}+\frac{7}{32} \xi\right)\right. \\
\left.+\frac{599}{32}-\frac{3}{4} \zeta_{3}-\frac{9}{64} \xi+\frac{3}{8} \xi^{2}-\frac{3}{16} \xi^{2} \zeta_{3}\right\}+\mathcal{O}(\varepsilon) .
\end{array}
$$

\section{$7 \quad$ WST identity at the two-loop level}

Due to the differential WST identity, we get the representations (3.7) and (3.8) for the functions $T_{i}\left(p^{2}\right)$. In the massless case, all one-loop expressions are proportional to $\left(p^{2}\right)^{-\varepsilon}$, whereas two-loop expressions contain $\left(p^{2}\right)^{-2 \varepsilon}$. Thus, the differentiations in (3.8) become trivial. Expanding in $g^{2}$, we get]

$$
\begin{gathered}
T_{1}^{(1)}\left(p^{2}\right)=a_{3}^{(1)}\left(p^{2}\right)+G^{(1)}\left(p^{2}\right)+J^{(1)}\left(p^{2}\right), \\
T_{1}^{(2)}\left(p^{2}\right)=a_{3}^{(1)}\left(p^{2}\right)\left[G^{(1)}\left(p^{2}\right)+J^{(1)}\left(p^{2}\right)\right]+G^{(1)}\left(p^{2}\right) J^{(1)}\left(p^{2}\right) \\
+a_{3}^{(2)}\left(p^{2}\right)+G^{(2)}\left(p^{2}\right)+J^{(2)}\left(p^{2}\right), \\
T_{2}^{(1)}\left(p^{2}\right)=2 T_{1}^{(1)}\left(p^{2}\right)-2\left[(1-\varepsilon) J^{(1)}\left(p^{2}\right)+(1+\varepsilon) a_{2}^{(1)}\left(p^{2}\right)+\widetilde{a}_{2}^{(1)}\left(p^{2}\right)\right],
\end{gathered}
$$

\footnotetext{
${ }^{8}$ We take into account that (in the massless case) $G(0)=1$.
} 


$$
\begin{aligned}
T_{2}^{(2)}\left(p^{2}\right)= & 2 T_{1}^{(2)}\left(p^{2}\right)-2\left[J^{(1)}\left(p^{2}\right) a_{2}^{(1)}\left(p^{2}\right)+J^{(1)}\left(p^{2}\right) \widetilde{a}_{2}^{(1)}\left(p^{2}\right)\right. \\
& \left.+(1-2 \varepsilon) J^{(2)}\left(p^{2}\right)+(1+2 \varepsilon) a_{2}^{(2)}\left(p^{2}\right)+\widetilde{a}_{2}^{(2)}\left(p^{2}\right)\right] .
\end{aligned}
$$

Substituting the expressions for ghost-gluon vertex and two-point functions, we arrive at the same results as given in (4.8)-(4.11).

\section{Renormalization}

To begin this section, we would like to explain why the zero-momentum limit of the threegluon vertex, as well as the relevant limits of the ghost-gluon vertex, are infrared finite, i.e. we do not get any $1 / \varepsilon$ poles of infrared (on-shell) origin. The main argument is just power counting.

Consider a triple vertex $V_{0}$ (part of a two-loop diagram) to which are attached the zero-momentum external line, together with two adjacent propagators carrying the same loop momentum $q$. In the case of a scalar (say, $\phi^{3}$ ) theory, one would get $1 /\left(q^{2}\right)^{2}$ in the integrand, leading to an infrared divergency. However, in QCD the vertex $V_{0}$ can be either (i) a three-gluon vertex, (ii) a ghost-gluon vertex, or (iii) a quark-gluon vertex. Effectively, the power of the gluon or ghost propagator in QCD is $1 /\left(q^{2}\right)$, whereas for the massless quark propagator we get $1 / q$. Therefore, the case (iii) is infrared finite, since we get only $1 / q^{2}$ from the two quark propagators (no $q$-dependent factor from the vertex). In the cases (i) and (ii), we get $1 /\left(q^{2}\right)^{2}$ from the two gluon (or ghost) propagators. However, we also get a momentum-dependent factor from the three-gluon (or ghost-gluon) vertex $V_{0}$, which cannot contain any momentum other than $q$ (since the external momentum is zero). This gives in the numerator a factor which is linear in $q$, so that effectively the infrared behaviour is just $1 / q^{3}$, i.e. we have no infrared divergency. When the zero-momentum line is attached to the four-gluon vertex like e.g. in diagrams $(h)$ and $\left(h^{\prime}\right)$ in Fig. 1, we may also get two propagators carrying the same momentum $q$. However, a similar power counting shows that there are no infrared singularities. For example, in diagrams $(h)$ and $\left(h^{\prime}\right)$ an extra momentum $q$ appears in the numerator from the one-loop self-energy-type insertion. This explains why all singularities in this limit are of ultraviolet origin, and therefore should be removed by renormalization.

In this paper we adopt the modification of the renormalization prescription by ' $t$ Hooft [27], corresponding to the so-called $\overline{\mathrm{MS}}$ scheme [28]. In this section (and in Appendix B), the notations $\xi, \alpha, g^{2}$, etc. (without subscript) correspond to the renormalized (in the $\overline{\mathrm{MS}}$ scheme) quantities. In previous sections (and in Appendix A), they should be understood as the bare quantities $\xi_{B}, \alpha_{B}, g_{B}^{2}$, etc.

The renormalization constants $Z_{\Gamma}$ relating the dimensionally-regularized one-particleirreducible Green functions to the renormalized ones,

$$
\Gamma^{(\mathrm{ren})}\left(\left\{\frac{p_{i}^{2}}{\mu^{2}}\right\}, \alpha, g^{2}\right)=\lim _{\varepsilon \rightarrow 0}\left[Z_{\Gamma}\left(\frac{1}{\varepsilon}, \alpha, g^{2}\right) \Gamma\left(\left\{p_{i}^{2}\right\}, \alpha_{B}, g_{B}^{2}, \varepsilon\right)\right],
$$

look in this scheme like

$$
Z_{\Gamma}\left(\frac{1}{\varepsilon}, \alpha, g^{2}\right)=1+\sum_{j=1}^{\infty} C_{\Gamma}^{[j]}\left(\alpha, g^{2}\right) \frac{1}{\varepsilon^{j}}
$$


where $\alpha=1-\xi$. In eq. (8.1) $\mu$ is the renormalization parameter with the dimension of mass. It is assumed that on the r.h.s. of eq. (8.1) the squared bare charge $g_{B}^{2}$ and the bare gauge parameter $\alpha_{B}$ must be substituted in terms of renormalized ones, multiplied by appropriate $Z$ factors (cf. eqs. (8.8) and (8.9)).

We use the following definitions for renormalization factors:

$$
\begin{aligned}
& \Gamma_{\mu_{1} \mu_{2} \mu_{3}}^{(\text {ren }}\left(p_{1}, p_{2}, p_{3}\right)=Z_{1} \Gamma_{\mu_{1} \mu_{2} \mu_{3}}\left(p_{1}, p_{2}, p_{3}\right), \\
& \Pi_{\mu_{1} \mu_{2}}^{\left(\text {ren } a_{1} a_{2}\right.}(p)=Z_{3} \Pi_{\mu_{1} \mu_{2}}^{a_{1} a_{2}}(p), \\
& \widetilde{\Gamma}_{\mu}^{\left(\text {ren } a_{1} a_{2} a_{3}\right.}\left(p_{1}, p_{2}, p_{3}\right)=\widetilde{Z}_{1} \tilde{\Gamma}_{\mu}^{a_{1} a_{2} a_{3}}\left(p_{1}, p_{2}, p_{3}\right), \\
& \widetilde{\Pi}^{\text {(ren }) a_{1} a_{2}}\left(p^{2}\right)=\widetilde{Z}_{3} \widetilde{\Pi}^{a_{1} a_{2}}\left(p^{2}\right),
\end{aligned}
$$

where $\Pi_{\mu_{1} \mu_{2}}^{a_{1} a_{2}}(p)$ and $\widetilde{\Pi}^{a_{1} a_{2}}\left(p^{2}\right)$ are the gluon polarization operator and the ghost self energy, respectively. For the scalar amplitudes, eqs. (8.5)-(8.6) mean that $J\left(p^{2}\right)$ and $G\left(p^{2}\right)$ should be renormalized by means of $Z_{3}$ and $\widetilde{Z}_{3}^{-1}$, respectively. Furthermore, according to eqs. (8.3)-(8.4) the three-gluon amplitudes $\left(T_{1}\right.$ and $\left.T_{2}\right)$ should be renormalized using $Z_{1}$, whereas for the ghost-gluon functions $\left(a_{3}, e_{3}, a_{2}\right.$ and $\left.e_{2}^{\prime}\right)$ one should use $\widetilde{Z}_{1}$.

The WST identity requires that

$$
\frac{Z_{3}}{Z_{1}}=\frac{\widetilde{Z}_{3}}{\widetilde{Z}_{1}}
$$

If this condition is satisfied, the WST identity is valid for the renormalized quantities, too.

Using (8.7), the bare coupling constant $g_{B}^{2}$ can be chosen (in the $\overline{\mathrm{MS}}$ scheme) as?

$$
g_{B}^{2}=\left(\frac{\mu^{2} e^{\gamma}}{4 \pi}\right)^{\varepsilon} g^{2} \widetilde{Z}_{1}^{2} Z_{3}^{-1} \widetilde{Z}_{3}^{-2}=\left(\frac{\mu^{2} e^{\gamma}}{4 \pi}\right)^{\varepsilon} g^{2} Z_{1}^{2} Z_{3}^{-3} .
$$

The gauge parameter $\alpha=1-\xi$ is renormalized as

$$
\alpha_{B}=Z_{3} \alpha, \quad \text { so that } \quad \xi_{B}=1-Z_{3}(1-\xi) .
$$

Below we shall use the following notation:

$$
h \equiv \frac{g^{2}}{(4 \pi)^{2}}=\frac{\alpha_{s}}{4 \pi}, \quad \text { where } \quad \alpha_{s} \equiv \frac{g^{2}}{4 \pi} .
$$

The two-loop-order results for the renormalization factors have been obtained in 10 , 11, 12] (see also in ref. [26]). For completeness, we list the corresponding expressions in Appendix B.

Using eqs. (4.1)-(4.4), (4.8)-(4.11), (8.3) and (B.1), we obtain the renormalized scalar amplitudes appearing in the three-gluon vertex (cf. eq. (2.3)),

$$
\begin{aligned}
T_{1}^{(\text {ren })}= & +h\left[C_{A}\left(-\frac{35}{18}+\frac{1}{2} \xi-\frac{1}{4} \xi^{2}\right)+\frac{20}{9} T\right] \\
+ & h^{2}\left[C_{A}^{2}\left(-\frac{4021}{288}-\frac{1}{4} \zeta_{3}-\frac{2317}{576} \xi+\frac{15}{8} \xi \zeta_{3}+\frac{113}{144} \xi^{2}-\frac{1}{16} \xi^{3}+\frac{1}{16} \xi^{4}\right)\right. \\
& \left.+C_{A} T\left(\frac{875}{72}+8 \zeta_{3}+\frac{20}{9} \xi-\frac{10}{9} \xi^{2}\right)+C_{F} T\left(\frac{55}{3}-16 \zeta_{3}\right)\right]+\mathcal{O}\left(h^{3}\right),
\end{aligned}
$$

\footnotetext{
${ }^{9}$ The factor $\left(e^{\gamma} /(4 \pi)\right)^{\varepsilon}=\exp [\varepsilon(\gamma-\ln (4 \pi))]$ in eq. (3.8) represents the difference between the $\overline{\mathrm{MS}}$ and
} MS schemes (cf. also eq. (4.7)). 


$$
\begin{array}{r}
T_{2}^{(\text {ren })}=h\left[C_{A}\left(-\frac{4}{3}-2 \xi+\frac{1}{4} \xi^{2}\right)+\frac{8}{3} T\right]+h^{2}\left[C_{A} T\left(\frac{157}{9}-\frac{37}{18} \xi-\frac{2}{9} \xi^{2}\right)+8 C_{F} T\right. \\
\left.+C_{A}^{2}\left(-\frac{641}{36}-\zeta_{3}+\frac{5}{18} \xi-\frac{1}{2} \xi \zeta_{3}-\frac{287}{144} \xi^{2}+\frac{19}{16} \xi^{3}-\frac{1}{8} \xi^{4}\right)\right]+\mathcal{O}\left(h^{3}\right) .
\end{array}
$$

Here and henceforth, we put $p^{2}=-\mu^{2}$ in the renormalized expressions. In Feynman gauge $(\xi=0)$, our expressions agree with eq. (B4) from [8]. However, the one-loop part of the result for $T_{2}$ in an arbitrary (non-Feynman) gauge disagrees with eq. (A10) from [8]ण.

The renormalized expressions for two-point functions are

$$
\begin{aligned}
& J^{(\mathrm{ren})}=1+ h\left[C_{A}\left(-\frac{31}{9}+\xi-\frac{1}{4} \xi^{2}\right)+\frac{20}{9} T\right] \\
&+ h^{2}\left[C_{A}^{2}\left(-\frac{3245}{144}+\zeta_{3}-\frac{287}{96} \xi+2 \xi \zeta_{3}+\frac{61}{72} \xi^{2}-\frac{3}{16} \xi^{3}+\frac{1}{16} \xi^{4}\right)\right. \\
&+\left.C_{A} T\left(\frac{451}{36}+8 \zeta_{3}+\frac{10}{3} \xi-\frac{10}{9} \xi^{2}\right)+C_{F} T\left(\frac{55}{3}-16 \zeta_{3}\right)\right]+\mathcal{O}\left(h^{3}\right), \\
& G^{(\mathrm{ren})}=1+h C_{A}+h^{2}\left[C_{A}^{2}\left(\frac{997}{96}-\frac{3}{4} \zeta_{3}-\frac{41}{64} \xi+\frac{3}{8} \xi^{2}-\frac{3}{16} \xi^{2} \zeta_{3}\right)-\frac{95}{24} C_{A} T\right]+\mathcal{O}\left(h^{3}\right) .
\end{aligned}
$$

In Feynman gauge, eq. (8.13) gives the same as the first of eqs. (B3) in ref. [8]. Taking into account that

$$
\left[G^{-1}\right]^{(\mathrm{ren})}=2-G^{(\mathrm{ren})}+h^{2} C_{A}^{2}+\mathcal{O}\left(h^{3}\right),
$$

we have also confirmed the second of eqs. (B3) in [8], i.e. the result for the ghost self energy in Feynman gauge.

The renormalized expressions for the scalar functions occurring in the ghost-gluon vertex are

$$
\begin{aligned}
a_{3}^{(\text {ren })}= & 1+\frac{1}{2} h C_{A}(1-\xi) \\
& +h^{2}\left[C_{A}^{2}\left(\frac{137}{48}-\frac{1}{2} \zeta_{3}-\frac{299}{96} \xi-\frac{1}{8} \xi \zeta_{3}+\frac{7}{16} \xi^{2}+\frac{3}{16} \xi^{2} \zeta_{3}\right)+\frac{1}{4} C_{A} T\right]+\mathcal{O}\left(h^{3}\right),(8.16) \\
p^{2} e_{3}^{(\text {ren })}= & \frac{1}{4} h C_{A}(2+\xi)+h^{2}\left[C_{A}^{2}\left(\frac{20}{3}+\frac{1}{8} \zeta_{3}-\frac{5}{12} \xi+\frac{5}{16} \xi \zeta_{3}-\frac{3}{16} \xi^{2}\right)-\frac{8}{3} C_{A} T\right]+\mathcal{O}\left(h^{3}\right), \\
a_{2}^{(\text {ren })}= & 1+\frac{1}{4} h C_{A} \xi(1-\xi) \\
+ & h^{2}(1-\xi)\left[C_{A}^{2}\left(\frac{167}{72}-\zeta_{3}-\frac{43}{144} \xi-\frac{1}{16} \xi^{2}-\frac{1}{16} \xi^{3}\right)-\frac{5}{9} C_{A} T(1-\xi)\right]+\mathcal{O}\left(h^{3}\right),(8.18) \\
p^{2} e_{2}^{\prime(\text { ren })} & =\frac{1}{4} h C_{A}(1-\xi)(2-\xi) \\
+ & h^{2}(1-\xi)\left[C_{A}^{2}\left(\frac{29}{36}+\frac{5}{8} \zeta_{3}-\frac{5}{36} \xi-\frac{3}{16} \xi \zeta_{3}+\frac{1}{16} \xi^{2}+\frac{1}{16} \xi^{3}\right)+\frac{5}{9} C_{A} T(1-\xi)\right]+\mathcal{O}\left(h^{3}\right) . \quad(8.19)
\end{aligned}
$$
in [8]. 
We note that these functions are in the following correspondence with the functions $G_{1,2}\left(p^{2}\right)$ used in [8], eq. (A3):

$$
a_{3}+p^{2} e_{3} \leftrightarrow 1+G_{2}, \quad a_{2}+p^{2} e_{2}^{\prime} \leftrightarrow 1+G_{1}
$$

Using this connection, we have confirmed the two-loop-order results for $G_{1}$ and $G_{2}$ in the Feynman gauge, eq. (B5) of ref. [8], as well as the one-loop-order results for $G_{1}$ and $G_{2}$ in an arbitrary covariant gauge, eq. (A11) of [8].

\section{Conclusion}

In the limit when one of the gluon momenta vanishes, we have calculated the two-loop contributions to the three-gluon vertex, in an arbitrary covariant gauge. In fact, we needed to calculate two scalar functions, $T_{1}\left(p^{2}\right)$ and $T_{2}\left(p^{2}\right)$, associated with different tensor structures, cf. eq. (2.3). Two independent ways of calculating these scalar functions have been realized. One of them is based on the straightforward calculation of all diagrams contributing to the two-loop three-gluon vertex shown in Fig. 1.

Another way of determining $T_{1}\left(p^{2}\right)$ and $T_{2}\left(p^{2}\right)$ is based on exploiting the differential WST identity (3.2). In this way, we obtain representations of the scalar functions $T_{1}\left(p^{2}\right)$ and $T_{2}\left(p^{2}\right)$, eqs. (3.7) and (3.8), in terms of the functions occurring in the ghost-gluon vertex (Fig. 2), its derivative (3.3), the gluon polarization operator (Fig. 3) and the ghost propagator (cf. Fig. 4). We have calculated all these functions and confirmed the result of the straightforward calculation.

The construction of the differential WST identity is of a certain interest, since in this limit it completely defines the three-gluon vertex, without leaving any "undetected" transverse contributions.

We have constructed renormalized expressions for all Green functions involved. Note that in the zero-momentum limit the three-gluon vertex has no infrared (on-shell) singularities, this is a "pure" case for performing the ultraviolet renormalization.

The obtained results can be considered as the first step in constructing expressions for the QCD vertices in more complicated cases, including on-shell configurations and the general off-shell case. In principle, the techniques for calculating the corresponding scalar integrals are already available [29, 30].

Acknowledgements. We are grateful to S.A. Larin for useful discussions. O. T. would like to thank Department of Physics, University of Bergen for warm hospitality during his visit in 1996, when this work was started. P. O. would like to thank the DESY Theory group, where this work was finished, for kind hospitality. This research has been supported by the Research Council of Norway, and by the Nordic project (NORDITA) 'Fundamental constituents of matter'.

\section{Appendix A: One-loop expressions for arbitrary $n$}

At the zero-loop level, we have

$$
a_{3}^{(0)}=a_{2}^{(0)}=1, \quad \widetilde{a}_{2}^{(0)}=0, \quad d_{2}^{(0)}=0, \quad J^{(0)}=G^{(0)}=1
$$


and the r.h.s. of eq. (3.2) restores the zero-loop result for the three-gluon vertex,

$$
\Gamma_{\mu_{1} \mu_{2} \mu_{3}}^{(0)}(p,-p, 0)=2 g_{\mu_{1} \mu_{2}} p_{\mu_{3}}-g_{\mu_{1} \mu_{3}} p_{\mu_{2}}-g_{\mu_{2} \mu_{3}} p_{\mu_{1}} \text {. }
$$

At the one-loop level, the expressions obtained in [6] give the following results in the zero-momentum limit:

$$
\begin{aligned}
& a_{3}^{(1)}\left(p^{2}\right)=\frac{g^{2} \eta}{(4 \pi)^{n / 2}} \frac{C_{A}}{4} \kappa\left(p^{2}\right)(n-2)(1-\xi), \\
& p^{2} e_{3}^{(1)}\left(p^{2}\right)=-\frac{g^{2} \eta}{(4 \pi)^{n / 2}} \frac{C_{A}}{8} \kappa\left(p^{2}\right)(n-4)[2+(n-3) \xi], \\
& a_{2}^{(1)}\left(p^{2}\right)=\frac{g^{2} \eta}{(4 \pi)^{n / 2}} \frac{C_{A}}{8} \kappa\left(p^{2}\right)(1-\xi)[4(n-3)-(n-4) \xi], \\
& p^{2} d_{2}^{(1)}\left(p^{2}\right)=\frac{g^{2} \eta}{(4 \pi)^{n / 2}} \frac{C_{A}}{8} \kappa\left(p^{2}\right)\left[2(n-6)-(5 n-18) \xi+(n-4) \xi^{2}\right], \\
& p^{2} e_{2}^{\prime(1)}\left(p^{2}\right)=-\frac{g^{2} \eta}{(4 \pi)^{n / 2}} \frac{C_{A}}{8} \kappa\left(p^{2}\right)(1-\xi)(2-\xi)(n-4), \\
& \widetilde{a}_{2}^{(1)}\left(p^{2}\right)=\frac{g^{2} \eta}{(4 \pi)^{n / 2}} \frac{C_{A}}{32} \kappa\left(p^{2}\right)\left\{8\left(n^{2}-6 n+10\right)-2 \xi\left(3 n^{2}-26 n+52\right)+\xi^{2}(n-4)(n-6)\right\} .
\end{aligned}
$$

In these equations,

$$
\kappa\left(p^{2}\right) \equiv-\frac{2}{(n-3)(n-4)}\left(-p^{2}\right)^{(n-4) / 2}=\frac{1}{\varepsilon(1-2 \varepsilon)}\left(-p^{2}\right)^{-\varepsilon} .
$$

The results for two-point functions are (cf. e.g. in [25, 6]):

$$
\begin{aligned}
& J^{(1)}\left(p^{2}\right)=\frac{g^{2} \eta}{(4 \pi)^{n / 2}} \frac{\kappa\left(p^{2}\right)}{(n-1)}\left\{-\frac{C_{A}}{8}[4(3 n-2)+4(n-1)(2 n-7) \xi-\right.\left.(n-1)(n-4) \xi^{2}\right] \\
&++2 T(n-2)\} \\
& G^{(1)}\left(p^{2}\right)=\frac{g^{2} \eta}{(4 \pi)^{n / 2}} \frac{C_{A}}{4} \kappa\left(p^{2}\right)[2+(n-3) \xi] .
\end{aligned}
$$

Taking into account that

$$
\begin{aligned}
{\left[\left(a_{2}-p^{2} d_{2}\right) J\right]^{(1)} } & =a_{2}^{(1)}-p^{2} d_{2}^{(1)}+J^{(1)} \\
{\left[\left(p^{2} d_{2}+\widetilde{a}_{2}-p^{2} \frac{\mathrm{d} a_{2}}{\mathrm{~d} p^{2}}\right) J+p^{2} a_{2} \frac{\mathrm{d} J}{\mathrm{~d} p^{2}}\right]^{(1)} } & =p^{2} d_{2}^{(1)}+\widetilde{a}_{2}^{(1)}-p^{2} \frac{\mathrm{d} a_{2}^{(1)}}{\mathrm{d} p^{2}}+p^{2} \frac{\mathrm{d} J^{(1)}}{\mathrm{d} p^{2}} \\
& =p^{2} d_{2}^{(1)}+\widetilde{a}_{2}^{(1)}-\frac{n-4}{2} a_{2}^{(1)}+\frac{n-4}{2} J^{(1)}
\end{aligned}
$$

we have checked that eq. (3.2) is satisfied at the one-loop level, for an arbitrary $n$. Furthermore,

$$
a_{2}^{(1)}\left(p^{2}\right)-p^{2} d_{2}^{(1)}\left(p^{2}\right)=a_{3}^{(1)}\left(p^{2}\right)+G^{(1)}\left(p^{2}\right)=\frac{g^{2} \eta}{(4 \pi)^{n / 2}} \frac{C_{A}}{4} \kappa\left(p^{2}\right)(n-\xi) .
$$

Therefore, eq. (3.5) (which follows from eq. (3.4)) is satisfied at the one-loop level. 


\section{Appendix B: Renormalization factors}

The expressions for the relevant two-loop-order renormalization factors have been presented in refs. 10, 11, 12] (cf. also in [26]). For completeness, we present the corresponding expressions herett:

$$
\begin{gathered}
Z_{1}=1+\frac{h}{\varepsilon}\left[C_{A}\left(\frac{2}{3}+\frac{3}{4} \xi\right)-\frac{4}{3} T\right]+h^{2}\left\{C_{A} T\left[\frac{1}{\varepsilon^{2}}\left(\frac{5}{2}-\xi\right)-\frac{25}{12 \varepsilon}\right]-\frac{2}{\varepsilon} C_{F} T\right. \\
\left.+C_{A}^{2}\left[\frac{1}{\varepsilon^{2}}\left(-\frac{13}{8}-\frac{7}{16} \xi+\frac{15}{32} \xi^{2}\right)+\frac{1}{\varepsilon}\left(\frac{71}{48}+\frac{45}{32} \xi-\frac{3}{16} \xi^{2}\right)\right]\right\}+\mathcal{O}\left(h^{3}\right), \\
\widetilde{Z}_{1}=1-\frac{h}{2 \varepsilon} C_{A}(1-\xi)+h^{2} C_{A}^{2}(1-\xi)\left[\frac{1}{\varepsilon^{2}}\left(\frac{5}{8}-\frac{1}{4} \xi\right)+\frac{1}{\varepsilon}\left(-\frac{3}{8}+\frac{1}{16} \xi\right)\right]+\mathcal{O}\left(h^{3}\right), \\
Z_{3}=1+\frac{h}{\varepsilon}\left[C_{A}\left(\frac{5}{3}+\frac{\xi}{2}\right)-\frac{4}{3} T\right]+h^{2}\left\{C_{A} T\left[\frac{1}{\varepsilon^{2}}\left(\frac{5}{3}-\frac{2}{3} \xi\right)-\frac{5}{2 \varepsilon}\right]-\frac{2}{\varepsilon} C_{F} T\right. \\
\left.+C_{A}^{2}\left[\frac{1}{\varepsilon^{2}}\left(-\frac{25}{12}+\frac{5}{24} \xi+\frac{1}{4} \xi^{2}\right)+\frac{1}{\varepsilon}\left(\frac{23}{8}+\frac{15}{16} \xi-\frac{1}{8} \xi^{2}\right)\right]\right\}+\mathcal{O}\left(h^{3}\right), \\
\widetilde{Z}_{3}=1+\frac{h}{\varepsilon} C_{A}\left(\frac{1}{2}+\frac{1}{4} \xi\right)+h^{2}\left\{C_{A}^{2}\left[\frac{1}{\varepsilon^{2}}\left(-1-\frac{3}{16} \xi+\frac{3}{32} \xi^{2}\right)+\frac{1}{\varepsilon}\left(\frac{49}{48}-\frac{1}{32} \xi\right)\right]\right. \\
\left.+C_{A} T\left(\frac{1}{2 \varepsilon^{2}}-\frac{5}{12 \varepsilon}\right)\right\}+\mathcal{O}\left(h^{3}\right),
\end{gathered}
$$

where $\varepsilon=(4-n) / 2$ and $h=g^{2} /(4 \pi)^{2}$. One can check that eqs. (B.1)-(B.4) obey the WST identity (8.7), so only three of them are independent. Using the results for unrenormalized Green functions, we have performed an independent check on these $Z$ factors 2 .

The results for these renormalization factors (without fermionic contributions, i.e. for the pure Yang-Mills theory) were first presented in [10] (Feynman gauge) and [11] (an arbitrary covariant gauge). The complete results in an arbitrary covariant gauge, including the fermionic contributions, were presented in [12] ( $\widetilde{\sim}_{\text {. }}$. also in [26]). In [12], the renormalization factors $Z_{3}$ and $\widetilde{Z}_{3}$ were denoted as $Z_{2}$ and $\widetilde{Z}_{2}$. There was an obvious misprint in the last term of the expression for $Z_{2}$ where $\frac{\alpha^{2}}{2} T^{2}$ should read $\frac{C_{2}}{2} t N$ (in their notation, $\left.T^{2} \leftrightarrow C_{F}, C_{2} \leftrightarrow C_{A}, t N \leftrightarrow T\right)$. We note that this misprint was copied over to the review [31] and the textbook [25]. In [25], in the end of the first line of eq. (C.6) for $\widetilde{Z}_{3}$, the term $\alpha_{R}^{2} C_{F}$ should read $C_{G} T_{R} N_{f}\left(\alpha_{R}\right.$ is the renormalized gauge parameter, $C_{G} \leftrightarrow C_{A}$ ). Then, in the beginning of the last line of eq. (C.5) for $Z_{3}, \frac{1}{8} C_{G}$ should read $\frac{1}{8} C_{G}^{2}$. There are several misprints in eq. (2.30b) of [31]. The term $\frac{\alpha_{G}^{2}}{2}\left(\frac{1}{4}\right) \frac{N^{2}-1}{2 N}$ should read $\frac{N}{2}\left(\frac{1}{4}\right) \frac{n}{2}$ ( $\alpha_{G}$ is the renormalized gauge parameter, $\left.n \leftrightarrow N_{f}, \frac{n}{2} \leftrightarrow T\right)$. In the previous term, $\frac{N}{4}$ should read $\frac{N^{2}}{4}$. In the term involving $\frac{5}{12}$, the "factor" $\frac{n}{8}$ with the following bracket should be removed. In the one-loop-order part, $\frac{\alpha_{G}}{3}$ should read $\frac{\alpha_{G}}{2}$,

\footnotetext{
${ }^{11}$ As in section 8 , the renormalized quantities $\xi=1-\alpha, g^{2}$, etc. are understood.

${ }^{12}$ Note that the two-loop results for $Z$ factors in the MS scheme are of the same form as in the $\overline{\mathrm{MS}}$ scheme; the only difference is that $g^{2}$ in the definition of $h$ should be understood as the renormalized squared charge in the MS scheme.
} 
cf. eq. (2.30a). Finally, in eq. (2.31b) for $\widetilde{Z}_{1}$, the one-loop-order contribution should be multiplied by $\frac{1}{4}$, cf. eq. (2.31a).

Using the $1 / \varepsilon$ term of the renormalization factor $Z_{\Gamma}$ (cf. eq. (8.2)), one can obtain the corresponding anomalous dimension $\gamma_{\Gamma}$ via

$$
\gamma_{\Gamma}\left(\alpha, g^{2}\right)=g^{2} \frac{\partial}{\partial g^{2}} C_{\Gamma}^{[1]}\left(\alpha, g^{2}\right)
$$

We have checked that in the Feynman gauge $\xi=0(\alpha=1)$ the results for the anomalous dimensions $\widetilde{\gamma}_{1}, \gamma_{3}$ and $\widetilde{\gamma}_{3}$ coincide (in the two-loop approximation) with those from [13]. The anomalous dimension $\gamma_{1}$ is related to the others via $\gamma_{1}-\gamma_{3}=\widetilde{\gamma}_{1}-\widetilde{\gamma}_{3}$ (this follows from the WST identity (8.7) and the definition (B.5)). Moreover, since (cf. in [13])

$$
\beta\left(g^{2}\right)=g^{2}\left[2 \widetilde{\gamma}_{1}\left(\alpha, g^{2}\right)-\gamma_{3}\left(\alpha, g^{2}\right)-2 \widetilde{\gamma}_{3}\left(\alpha, g^{2}\right)\right]
$$

we obtain the same result for the two-loop $\beta$ function as those given in $19,10,11$, 12$]^{[3}$, namely

$$
\frac{1}{g^{2}} \beta\left(g^{2}\right)=h\left[-\frac{11}{3} C_{A}+\frac{4}{3} T\right]+h^{2}\left[-\frac{34}{3} C_{A}^{2}+\frac{20}{3} C_{A} T+4 C_{F} T\right]+\mathcal{O}\left(h^{3}\right) .
$$

Higher terms of the $\beta$ function are available in refs. [13, 14, 15].

\section{References}

[1] W. Celmaster and R.J. Gonsalves, Phys. Rev. D20 (1979) 1420.

[2] P. Pascual and R. Tarrach, Nucl. Phys. B174 (1980) 123.

[3] J.S. Ball and T.-W. Chiu, Phys. Rev. D22 (1980) 2550; Erratum: D23 (1981) 3085.

[4] F.T. Brandt and J. Frenkel, Phys. Rev. D33 (1986) 464.

[5] M.A. Nowak, M. Praszałowicz and W. Słomiński, Ann. Phys. (N. Y.) 166 (1986) 443.

[6] A.I. Davydychev, P. Osland and O.V. Tarasov, Phys. Rev. D54 (1996) 4087.

[7] J.S. Ball and T.-W. Chiu, Phys. Rev. D22 (1980) 2542;

L.V. Dung, H.D. Phuoc and O.V. Tarasov, Sov. J. Nucl. Phys. 50 (1989) 1072;

A. Kızılersü, M. Reenders and M.R. Pennington, Phys. Rev. D52 (1995) 1242.

[8] E. Braaten and J.P. Leveille, Phys. Rev. D24 (1981) 1369.

[9] W.E. Caswell, Phys. Rev. Lett. 33 (1974) 244.

[10] D.R.T. Jones, Nucl. Phys. B75 (1974) 531.

\footnotetext{
${ }^{13}$ We just note two obvious misprints in $\left[10\right.$ : (i) in eq. (23), $B u^{2}$ should read $B u^{5}$ and (ii) in eq. (24) (one-loop-order part of the $\beta$ function) $\frac{8}{3} T(R)$ should read $\frac{4}{3} T(R)$. In eq. (4) of [9], the lower-case $z$ 's should be understood.
} 
[11] A.A. Vladimirov and O.V. Tarasov, Yad. Fiz. 25 (1977) 1104 [ Sov. J. Nucl. Phys. 25 (1977) 585 ].

[12] E.Sh. Egorian and O.V. Tarasov, Teor. Mat. Fiz. 41 (1979) 26 [ Theor. Math. Phys. 41 (1979) 863 ].

[13] O.V. Tarasov, A.A. Vladimirov and A.Yu. Zharkov, Phys. Lett. 93B (1980) 429.

[14] S.A. Larin and J.A.M. Vermaseren, Phys. Lett. B303 (1993) 334.

[15] T. van Ritbergen, J.A.M. Vermaseren and S.A. Larin, Phys. Lett. B400 (1997) 379.

[16] A.A. Slavnov, Teor. Mat. Fiz. 10 (1972) 153 [ Theor. Math. Phys. 10 (1972) 99 ];

J.C. Taylor, Nucl. Phys. B33 (1971) 436.

[17] W. Marciano and H. Pagels, Phys. Rep. 36 (1978) 137.

[18] G. 't Hooft and M. Veltman, Nucl. Phys. B44 (1972) 189;

C.G. Bollini and J.J. Giambiagi, Nuovo Cim. 12B (1972) 20.

[19] J.A.M. Vermaseren, Comput. Phys. Commun. 83 (1994) 45.

[20] P. Cvitanovic, Phys. Rev. D14 (1976) 1536.

[21] F.V. Tkachov, Phys. Lett. 100B (1981) 65;

K.G. Chetyrkin and F.V. Tkachov, Nucl. Phys. B192 (1981) 159.

[22] A.I. Davydychev, Phys. Lett. B263 (1991) 107.

[23] A.C. Hearn, REDUCE User's Manual (version 3.6), RAND publication CP78 (Santa Monica, 1995).

[24] J.A.M. Vermaseren, Symbolic Manipulation with FORM (Computer Algebra Nederland, Amsterdam, 1991).

[25] T. Muta, Foundations of Quantum Chromodynamics (World Scientific, Singapore, 1987).

[26] P. Pascual and R. Tarrach, QCD: Renormalization for the Practitioner, Springer, Berlin, 1984 (Lecture Notes in Physics, v.194).

[27] G. 't Hooft, Nucl. Phys. B61 (1973) 455.

[28] W.A. Bardeen, A.J. Buras, D.W. Duke and T. Muta, Phys. Rev. D18 (1978) 3998.

[29] R.J. Gonsalves, Phys. Rev. D28 (1983) 1542;

W.L. van Neerven, Nucl. Phys. B268 (1986) 453;

G. Kramer and B. Lampe, J. Math. Phys. 28 (1987) 945.

[30] N.I. Ussyukina and A.I. Davydychev, Phys. Lett. B298 (1993) 363; B332 (1994) 159; B348 (1995) 503.

[31] S. Narison, Phys. Rep. 84 (1982) 263. 


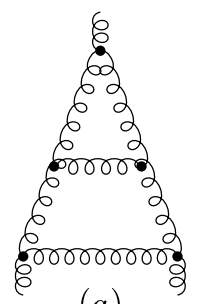

(a)
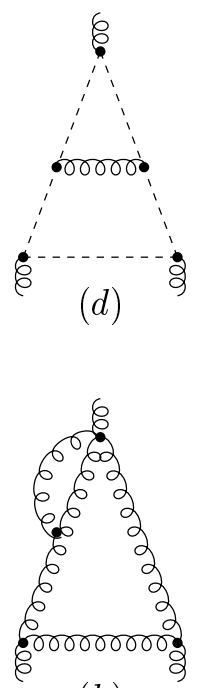

(h)

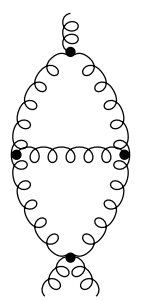

$(k)$

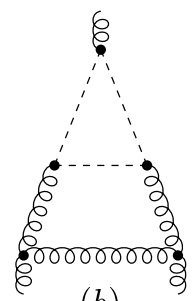

(b)
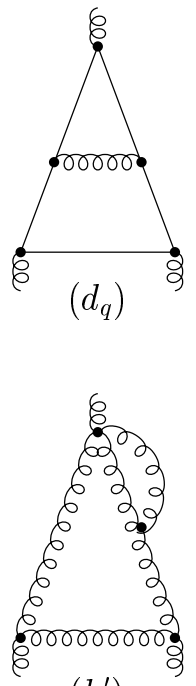

$\left(h^{\prime}\right)$

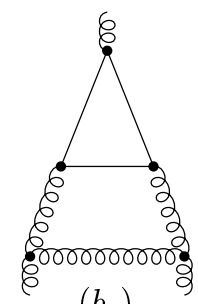

$\left(b_{q}\right)$

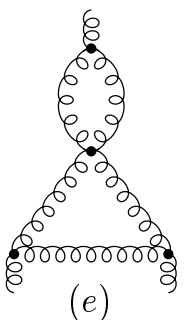

(e)

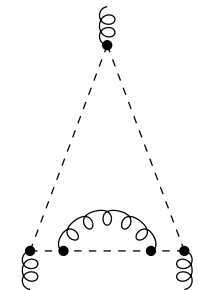

(i)

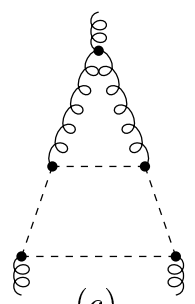

(c)
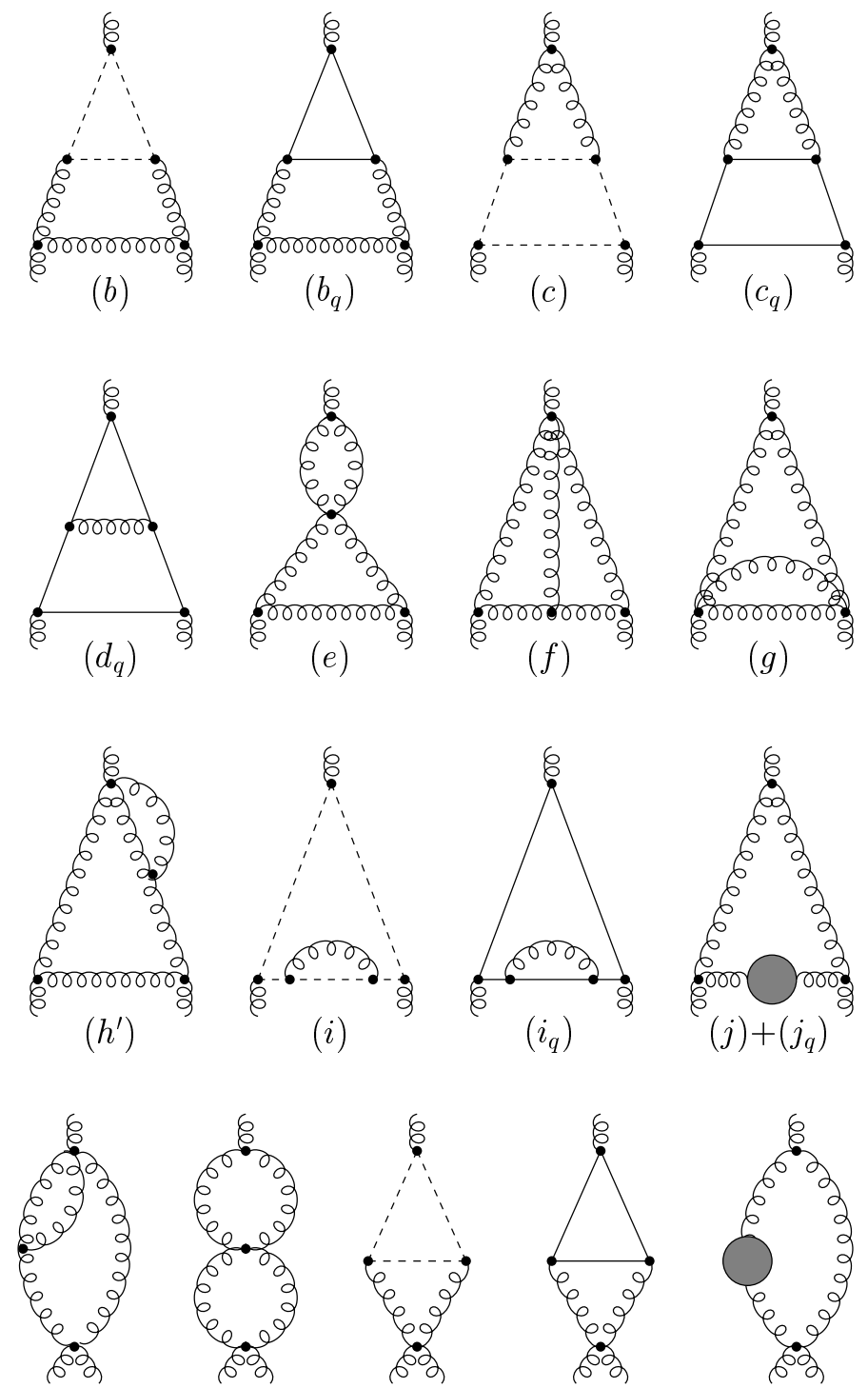

$(l)$

$(n)$

$\left(n_{q}\right)$

$(o)+\left(o_{q}\right)$

Figure 1: Two-loop three-gluon vertex diagrams. 

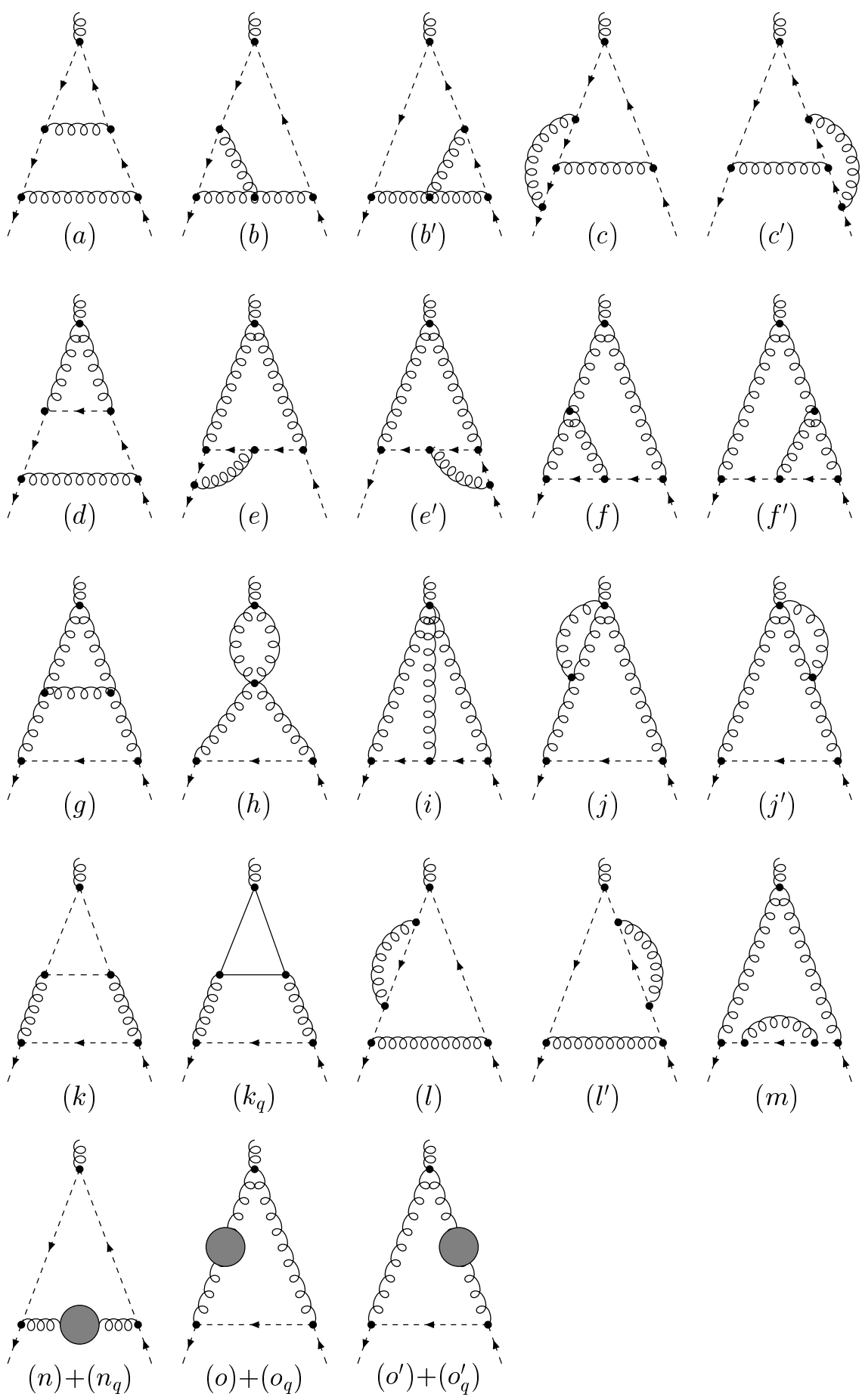

Figure 2: Two-loop ghost-gluon vertex diagrams. 


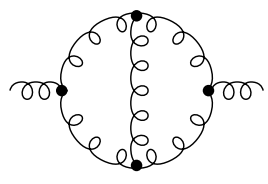

(a)

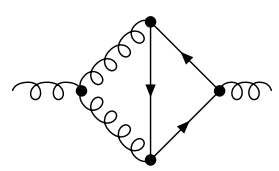

$\left(c_{q}\right)$

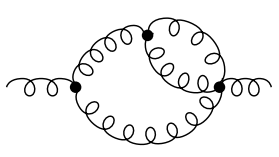

$(f)$

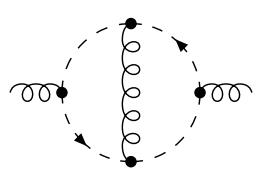

(b)

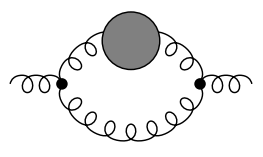

$$
(d)+\left(d_{q}\right)
$$

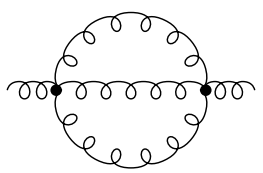

$(g)$

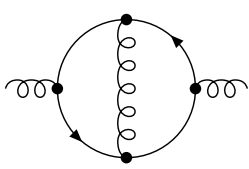

$\left(b_{q}\right)$

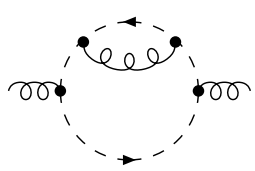

$(e)$

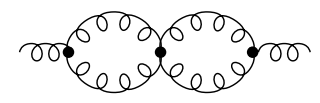

$(h)$

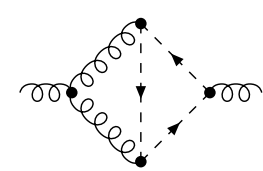

(c)

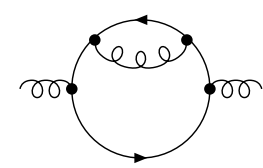

$\left(e_{q}\right)$

Figure 3: Two-loop gluon polarization operator diagrams.

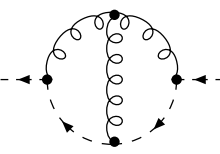

(a)

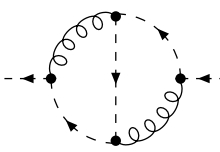

(b)

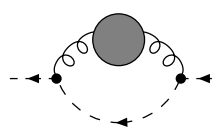

$(c)+\left(c_{q}\right)$

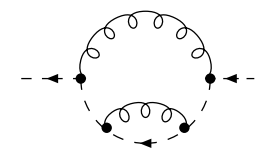

(d)

Figure 4: Two-loop ghost self-energy diagrams. 